\title{
Orthognathic Surgery for Correction of Facial Asymmetry after Condylar Fracture Using Computer Virtual Planning: A Case Report
}

\author{
Mohammed Nadershah
}

\begin{abstract}
Aim: This case report illustrates the management of post-traumatic malocclusion resulting from a condylar fracture during childhood using orthognathic surgery and three-dimensional virtual computer planning.

Background: Condylar fractures during childhood can result in severe functional and esthetic problems. The temporomandibular joint (TMJ) is the foundation for orthognathic surgery. The management of this complex dentofacial deformity represents a challenge to the oral and maxillofacial surgeon.

Case description: A 40-year-old man with a history of childhood trauma resulting in right sub-condylar fracture presented with severe facial asymmetry, canting of the occlusal plane, obstructive sleep apnea (OSA) symptoms, and limitation of his mouth opening. He underwent minimal presurgical orthodontics followed by orthognathic surgery including a LeFort 1 osteotomy, bilateral sagittal split osteotomy, and genioplasty. The occlusal plane was corrected by the counterclockwise rotation of the maxillomandibular complex (CCRMMC). A computer virtual planning facilitated the three-dimensional correction of this complex dentofacial deformity. It was possible to achieve the planned surgical movements despite difficulty in seating the condyle in the injured side.

Conclusion: Post-traumatic malocclusion resulting from a condylar fracture during childhood was managed by orthognathic surgery without TMJ surgery.

Clinical significance: Three-dimensional computer planning is a valuable aid in planning orthognathic surgery especially for facial asymmetry. Condylar seating during mandibular osteotomy is challenging even after years from condylar fracture. Temporomandibular joint surgery is not a necessity prior to orthognathic surgery in these cases if the patient has an acceptable mouth opening. The CCRMMC facilitated improvement in profile and OSA symptoms.
\end{abstract}

Keywords: Condylar fracture, Orthognathic surgery, Post-traumatic malocclusion, Virtual planning.

World Journal of Dentistry (2020): 10.5005/jp-journals-10015-1716

\section{INTRODUCTION}

Condylar fracture is a common injury affecting the facial skeleton. ${ }^{1}$ Nevertheless, the treatment of condylar fractures remains controversial. $^{2}$ The conventional treatment is by variable periods of maxillomandibular fixation (MMF) (closed reduction). The indications for open reduction and internal fixation are debatable in the literature. ${ }^{3}$ One of the main complications of condylar fractures in a growing child is the restriction of growth of the mandible on the affected side. This results in chin deviation, maxillary asymmetric growth, occlusal canting, development of obstructive sleep apnea (OSA), and limitation of the mouth opening. ${ }^{4}$

The correction of post-traumatic malocclusion and the associated dentofacial deformity represents a challenge for the treating surgeon and orthodontist. The temporomandibular joint (TMJ) is the foundation for orthognathic surgery. A malunited condylar fracture may require TMJ reconstruction with simultaneous or delayed orthognathic surgery. Alternative treatment options include selective occlusal grinding, camouflage orthodontics, and prosthodontic rehabilitation. However, none of these alternatives would correct the facial asymmetry.

The conventional surgical planning involves fabrication of surgical splints or guides in the dental lab using mounted casts. The mount relies on face-bow registration, dental casts, and bite registration. An advanced technology has recently resulted
Department of Oral and Maxillofacial Surgery, Faculty of Dentistry, King Abdulaziz University, Jeddah, Kingdom of Saudi Arabia

Corresponding Author: Mohammed Nadershah, Department of Oral and Maxillofacial Surgery, Faculty of Dentistry, King Abdulaziz University, Jeddah, Kingdom of Saudi Arabia, Phone: +966-12-6401000, e-mail: mnadershah@gmail.com

How to cite this article: Nadershah M. Orthognathic Surgery for Correction of Facial Asymmetry after Condylar Fracture Using Computer Virtual Planning: A Case Report. World J Dent 2020;11(2):156-160.

Source of support: Nil

Conflict of interest: None

in a paradigm shift in the planning of orthognathic surgery. A virtual planning begins with three-dimensional imaging of the facial skeleton. The dentition is incorporated into the facial scan by scanning the dental models or dentition using optic scanners or a cone beam computed tomography (CBCT) machine. Next, a computer software is used to process these images and simulate the surgery. Finally, a three-dimensional printer is used to fabricate intermediate and final surgical splints and cutting guides, if desired. We present a case of a malunited sub-condylar fracture that was treated with orthognathic surgery using virtual computer planning.

(O) The Author(s). 2020 Open Access This article is distributed under the terms of the Creative Commons Attribution 4.0 International License (https://creativecommons. org/licenses/by-nc/4.0/), which permits unrestricted use, distribution, and non-commercial reproduction in any medium, provided you give appropriate credit to the original author(s) and the source, provide a link to the Creative Commons license, and indicate if changes were made. The Creative Commons Public Domain Dedication waiver (http://creativecommons.org/publicdomain/zero/1.0/) applies to the data made available in this article, unless otherwise stated. 


\section{Case Description}

A 40-year-old man presented with a chief complaint of facial asymmetry that started after a childhood trauma to the lower jaw during a car accident. He sustained a right sub-condylar fracture when he was 5 years old. He did not receive any treatment for his injury and was managed by physical therapy. He does not have any other medical problems or surgical history. He denied taking any medications and he did not have any drug allergies. He reported daytime somnolence and snoring. He scored 19 on Epworth Sleepiness Scale which indicates severe daytime somnolence concerning for OSA. He was advised to get a sleep study, but he declined.

\section{Clinical Examination}

An extraoral clinical examination showed a significant deviation of the chin to the right. The nasal tip was slightly rotated to the right. The lower facial third height was reduced compared with middle and upper facial thirds. There was severe canting of the maxilla and the mandible. The gingival show during smiling at the upper left canine was $6 \mathrm{~mm}$ compared with $1 \mathrm{~mm}$ at the upper right canine. There was mild asymmetry at the cheek prominence levels. His profile was convex (Fig. 1). He had slight limitation in his mouth opening, and his maximal interincisal opening was $3 \mathrm{~cm}$. He did not have TMJ pain but had deviation of the mandible to the right with opening.

An intraoral clinical examination revealed multiple missing teeth: the upper right third molar, upper right second premolar, upper left second premolar, upper left second and third molars, lower left third molar, both lower central incisors, lower right second premolar, and lower right third molar. His occlusion demonstrated a crossbite in the right side and a scissor bite in the left side. Multiple teeth had restorations and crowns. He had a fair oral hygiene.

\section{Radiographic Examination}

Orthopantomogram (OPG) revealed an abnormally shaped right condyle (Fig. 2). Multiple teeth had previous root canal treatment. The right antigonial notch was more pronounced than the left one. A lateral cephalometric radiograph (Fig. 3) revealed high occlusal plane angle, convex profile, and retrognathic mandible. There were two levels of the inferior border of the mandible due to the severe canting. The posterior airway space at the level of the base of the tongue measured $1 \mathrm{~cm}$. Cone beam computed tomography showed an abnormally shaped right condyle.

\section{Treatment}

Presurgical orthodontics was started for leveling, alignment, and decompensation for about 6 months. The surgical movements were planned using computer virtual planning. The CBCT of the facial skeleton and of the dental casts in centric relation and final occlusion was uploaded via the internet to (3D Systems) company. Next, an online web meeting between myself and the computer engineer was held to plan the surgical movements. The surgical plan included a LeFort 1 osteotomy, bilateral sagittal split osteotomy, and genioplasty. The maxillomandibular complex was rotated in three dimensions to correct the cant and the mandibular deviation. The occlusal plan was also corrected by counterclockwise rotation of the complex to increase the facial projection and improve the posterior airway space (Fig. 4). The intermediate and final splints were generated using a three-dimensional printer. The details of the planned surgical movements are outlined in Table 1.

The mandibular movement was sequenced first due to the significant counterclockwise rotation of the maxillomandibular complex (CCRMMC). If the maxilla was sequenced first, this would result in a large intermediate splint anteriorly making it hard to seat the condyles intraoperatively. The bilateral sagittal split osteotomy was completed in a routine fashion. Extensive soft tissue stripping was completed to allow for this large movement. However, during the fixation of the mandible, it was difficult to seat the right condyle and rotate the proximal segment medially due to interferences at the abnormally shaped condylar head. A large gap between the right proximal and distal segments existed after the application of the titanium plates and screws. The decision was then to graft the gap with putty cortico-cancellous allograft. There was no difficulty in seating the condyle on the left side. The LeFort 1 was completed without any unexpected findings. Finally, an advancement and rotation genioplasty was completed without complications.

The patient was placed in MMF for 4 weeks. This decision was due to the minimal bone contact and the grafting between the right proximal and distal segments. He had a stable and reproducible occlusion When the MMF was released. He was placed on light guiding elastics for another 4 weeks. He was instructed to remain
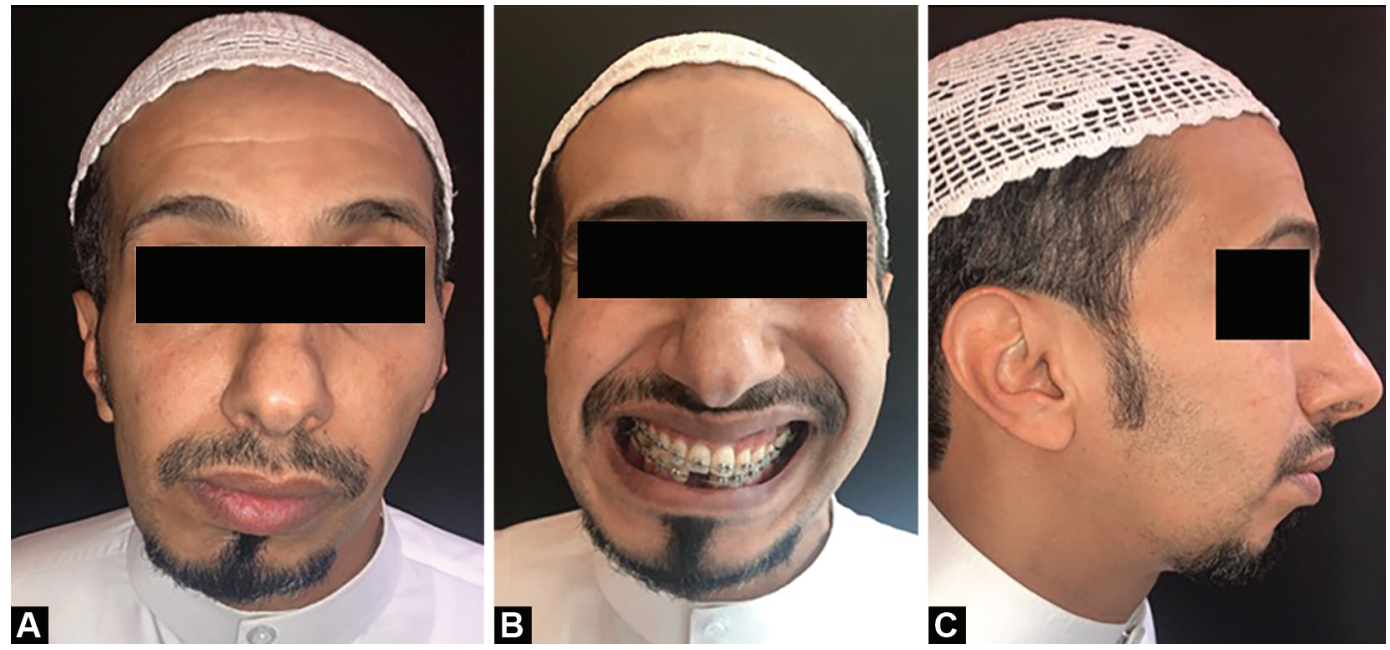

Figs $1 \mathrm{~A}$ to C: Presurgical extraoral pictures: (A) Frontal at rest; (B) During smiling; (C) Profile 


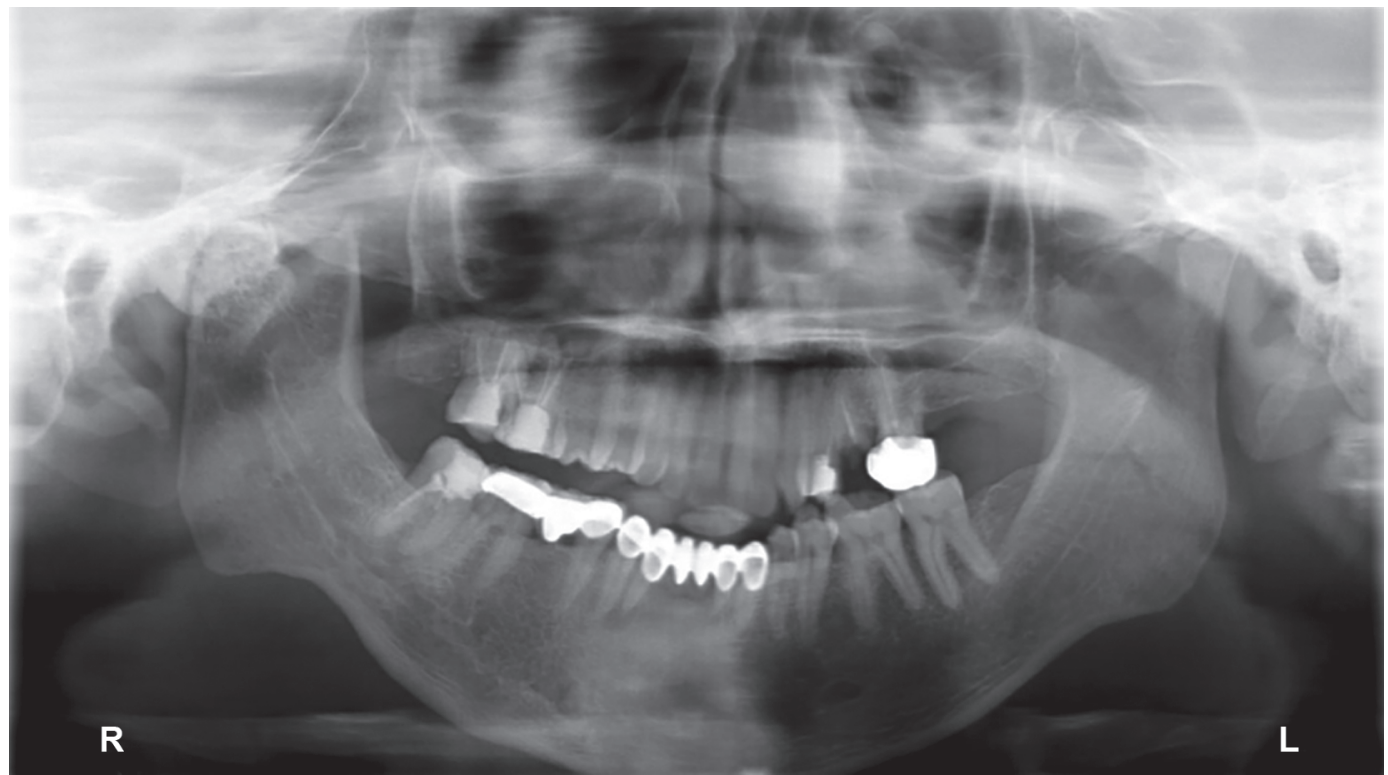

Fig. 2: Initial OPG demonstrating abnormally shaped right condyle and the accentuated right antigonial notch

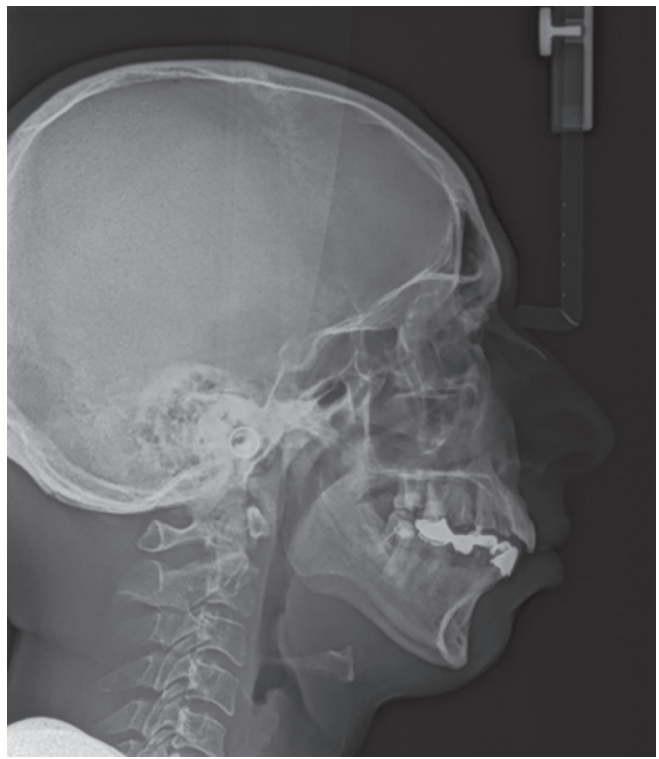

Fig. 3: Initial lateral cephalometric radiograph

on full liquid diet for 2 months after the surgery. His mouth opening was initially limited and improved to preoperative status with physical therapy. He also reported left TMJ pain in the first 2 months postoperatively, but it resolved gradually. His facial symmetry has greatly improved, and he was satisfied with the treatment outcome (Fig. 5). He did not show any signs of relapse at the 6-month follow-up. He reported that his snoring and daytime somnolence has resolved after the surgery.

\section{Discussion}

The ideal minimal time to wait after a condylar fracture before orthognathic surgery has not been established yet. The TMJ starts a remodeling phase within 2-3 weeks after condylar fractures and continues for several months. ${ }^{5,6}$ Becking et al. presented 21 patients treated by orthognathic surgery at least 9 months after the condylar process fracture with stable results. Only one patient had a complete relapse 3 months after using an inverted $L$ osteotomy to correct an anterior open bite. ${ }^{7}$ In addition, TMJ stability is important to allow for accurate positioning of the mandible during orthognathic surgery. Ellis suggested using imaging signs of cortication of the articular surfaces as a possible indication of the completion of the TMJ remodeling phase. ${ }^{8}$ The patient in this case report presented many years after the surgery and has established a functioning joint. Nevertheless, it was difficult to seat the right condyle during the mandibular fixation.

The mobility of the mandible must be assessed before pursuing orthognathic surgery. The limited range of motion of the mandible not only makes intraoral surgery difficult, but it may also worsen the hypomobility by adding the cicatricial effects of wound healing. ${ }^{9}$ Diminished TMJ mobility should be managed with aggressive physiotherapy to maximize mouth opening prior to surgical intervention. ${ }^{7}$ The reported patient had a slightly diminished mouth opening. However, he has been able to function well with that amount of mouth opening. An alternative treatment plan would include TMJ reconstruction (autogenous or prosthetic) prior to or simultaneously with orthognathic surgery.

Computer virtual planning in orthognathic surgery has recently gained popularity with proven accuracy in the literature. Schneider et al. reported improved accuracy and shorter operation time using virtual planning and pre-bent custom implants compared with the conventional planning. ${ }^{10}$ Zhang et al. compared the planned and actual results of orthognathic surgery using virtual computer planning. ${ }^{11}$ They reported a mean linear difference of $0.81 \mathrm{~mm}$ and mean angular difference of $0.95^{\circ}$. Ko et al. compared the surgical outcomes in patients with class III facial asymmetry using three-dimensional surgical planning vs conventional planning. ${ }^{12}$ They found a high degree of accuracy in results regardless of the experience of the surgeon. The main disadvantage of virtual computer planning is the high cost compared with the conventional planning.

The CCRMMC was reported to be an unstable movement possibly due to the resistance of the pterygomasseteric sling to lengthening of the posterior facial height. ${ }^{13}$ However, others found 

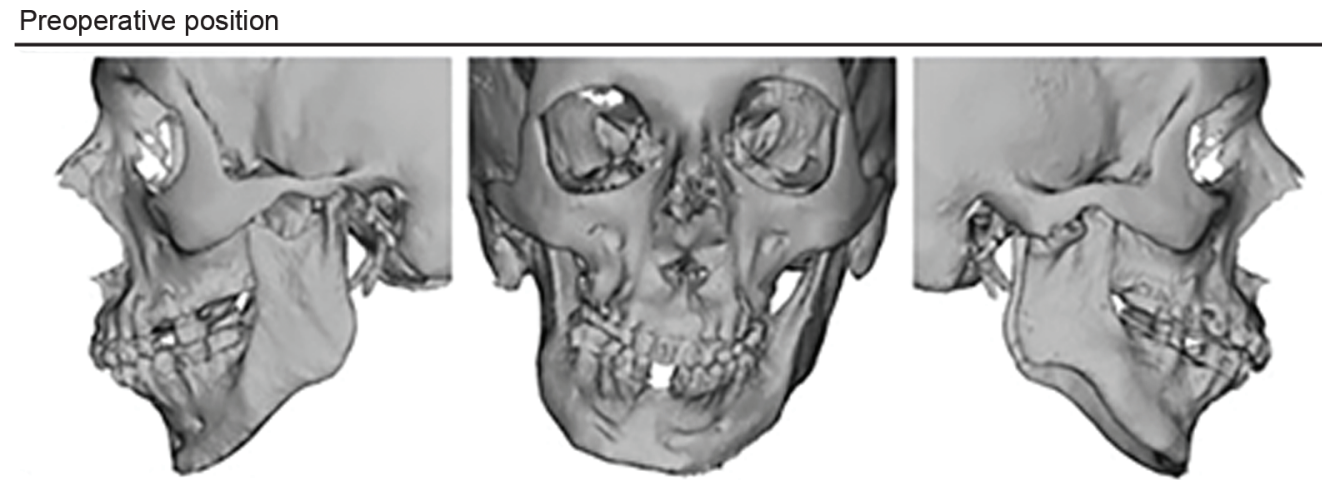

Intermediate position
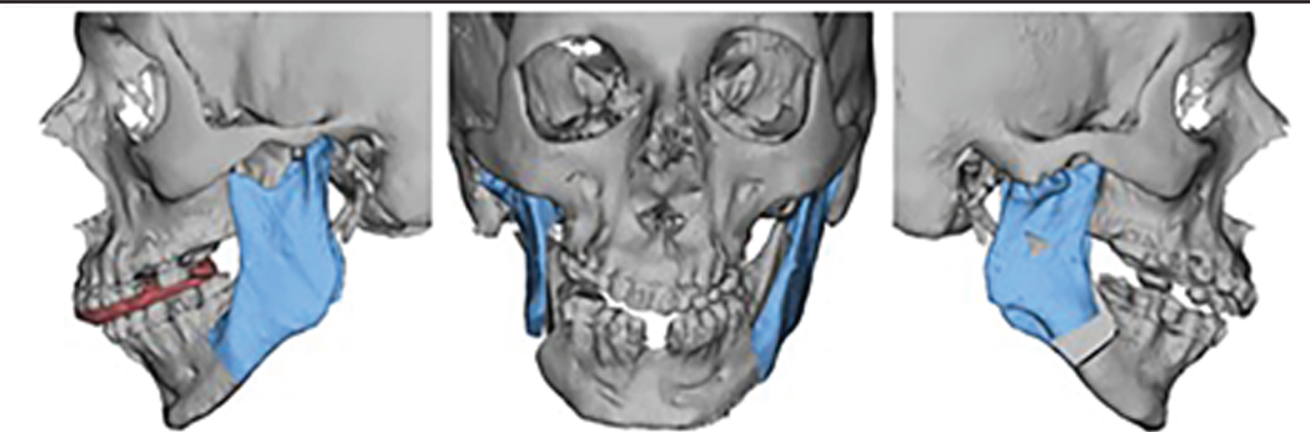

Postoperative position
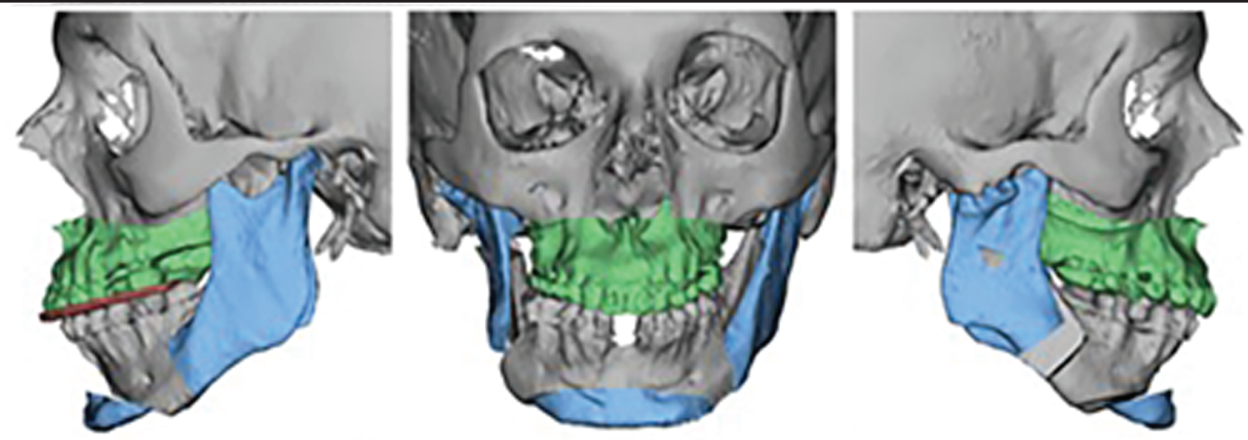

Fig. 4: The virtual three-dimensional surgical plan

Table 1: Details of the planned surgical movement for different points in the facial skeleton

\begin{tabular}{|c|c|c|c|c|c|c|}
\hline \multirow{2}{*}{$\begin{array}{l}\text { Point } \\
\text { Anterior nasal spine }\end{array}$} & \multicolumn{2}{|c|}{ Anterior/posterior (mm) } & \multicolumn{2}{|c|}{ Left/right $(\mathrm{mm})$} & \multicolumn{2}{|c|}{ Up/down $(\mathrm{mm})$} \\
\hline & 0.15 & Posterior & 1.10 & Right & 4.57 & Up \\
\hline A point & 0.74 & Anterior & 0.25 & Left & 4.33 & Up \\
\hline Midline of upper incisor & 3.00 & Anterior & 3.40 & Left & 4.25 & Up \\
\hline Upper left canine & 3.91 & Anterior & 3.80 & Left & 5.77 & Up \\
\hline Upper left first molar (mesiobuccal cusp) & 3.83 & Anterior & 3.97 & Left & 5.27 & Up \\
\hline Upper right canine & 1.42 & Anterior & 3.00 & Left & 1.08 & Up \\
\hline Upper right first molar (mesiobuccal cusp) & 0.19 & Anterior & 3.37 & Left & 2.62 & Down \\
\hline Midline of lower incisor & 3.20 & Anterior & 5.81 & Left & 3.38 & Up \\
\hline Lower left first molar (mesiobuccal cusp) & 2.34 & Anterior & 4.73 & Left & 5.01 & Up \\
\hline Lower right first molar (mesiobuccal cusp) & 2.76 & Anterior & 4.24 & Left & 3.13 & Down \\
\hline B point & 5.28 & Anterior & 8.66 & Left & 1.22 & Up \\
\hline Pogonion & 14.99 & Anterior & 15.69 & Left & 2.14 & Up \\
\hline
\end{tabular}

it to be a stable movement with adequate presurgical orthodontics and healthy TMJs. The mandible is a class III lever, and advancing the mandible will result in an increase in the mechanical load on the TMJ. ${ }^{14}$ Reyneke et al. found no difference in long-term stability of CCRMMC compared with clockwise rotation or no alteration in the occlusal plane. ${ }^{15}$ He recommended extensive stripping of 

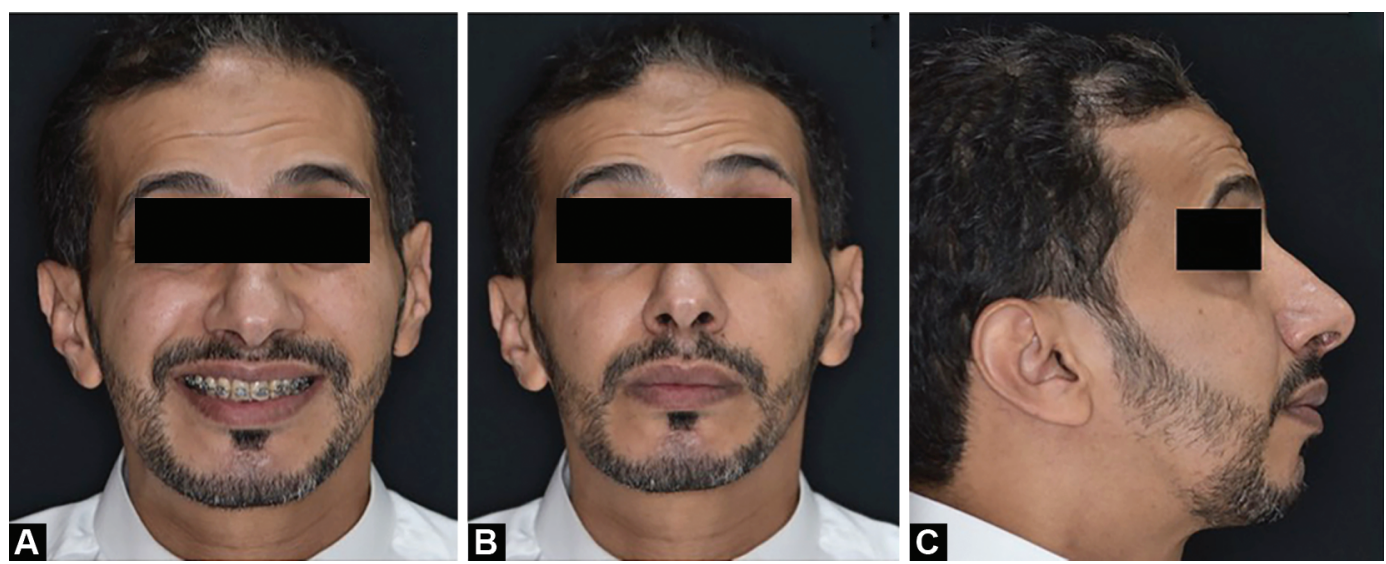

Figs 5A to C: Postsurgical pictures: (A) Frontal at rest, (B) During smiling; (C) Profile

the pterygomasseteric sling. This was done in our case. Mehra et al. reported that CCRMMC significantly improved the posterior airway space. ${ }^{16}$ Brevi et al. also reported the use of CCRMMC in the treatment of OSA. ${ }^{17}$ The patient in this case report subjectively reported improvement in his snoring and daytime somnolence after the surgery.

\section{CONCLUSION}

We presented a case of severe facial asymmetry secondary to condylar fracture during childhood that was managed by orthognathic surgery without TMJ surgery. A virtual computer planning facilitated successful treatment of the case. There was intraoperative difficulty in seating the fractured condyle despite the remote history of the trauma. The CCRMMC was used to improve facial esthetics and improve the posterior airway space.

\section{Declaration of Patient Consent}

The author certify that he has obtained all appropriate patient consent forms. In the form the patient has given his consent for his images and other clinical information to be reported in the journal. The patient understand that his name and initials will not be published, and due efforts will be made to conceal his identity, but anonymity cannot be guaranteed.

\section{References}

1. Zrounba H, Lutz J-C, Zink S, et al. Epidemiology and treatment outcome of surgically treated mandibular condyle fractures. A five years retrospective study. J Craniomaxillofac Surg 2014;42(6):879884. DOI: 10.1016/j.jcms.2014.01.002.

2. Rastogi S, Sharma S, Kumar S, et al. Fracture of mandibular condyleto open or not to open: an attempt to settle the controversy. Oral Surg Oral Med Oral Pathol Oral Radiol 2015;119(6):608-613. DOI: 10.1016/ j.00oo.2015.01.012.

3. Karan A, Kedarnath NS, Reddy GS, et al. Condylar FRactures: Surgical versus conservative management. Ann Maxillofac Surg 2019;9(1): 15-22. DOI: 10.4103/ams.ams_157_17.

4. Rocton S, Chaine A, Ernenwein D, et al. Mandibular fractures: epidemiology, therapeutic management, and complications in a series of 563 cases. Rev Stomatol Chir Maxillofac 2007;108(1):3-10; discussion 10-12. DOI: 10.1016/j.stomax.2006.11.001.

5. Chang S, Yang Y, Liu Y, et al. How does the remodeling capacity of children affect the morphologic changes of fractured mandibular condylar processes after conservative treatment? J Oral Maxillofac
Surg 2018;76(6):1279.e1-1279.e7. DOI: 10.1016/j.joms.2018. 01.029 .

6. Lindahl L, Hollender L. Condylar fractures of the mandible. II. a radiographic study of remodeling processes in the temporomandibular joint. Int J Oral Surg 1977;6(3):153-165. DOI: 10.1016/S0300-9785(77)80048-3.

7. Becking AG, Zijderveld SA, Tuinzing DB. The surgical management of post-traumatic malocclusion. Clin Plast Surg 2007;34(3):e37-e43. DOI: 10.1016/j.cps.2007.04.007.

8. Becking AG, Zijderveld SA, Tuinzing DB. Management of posttraumatic malocclusion caused by condylar process fractures. YJOMS 1998;56(12):1370-1374. ; discussion 1374-1375 10.1016/s02782391(98)90394-9.

9. Ellis E. Mobility of the mandible following advancement and maxillomandibular or rigid internal fixation: an experimental investigation in Macaca mulatta. YJOMS 1988;46(2):118-123. DOI: 10.1016/0278-2391(88)90262-5.

10. Schneider D, Kämmerer PW, Hennig $M$, et al. Customized virtual surgical planning in bimaxillary orthognathic surgery: a prospective randomized trial. Clin Oral Investig 2019;23(7):3115-3122. DOI: 10.1007/s00784-018-2732-3.

11. Zhang N, Liu S, Hu Z, et al. Accuracy of virtual surgical planning in two-jaw orthognathic surgery: comparison of planned and actual results. Oral Surg Oral Med Oral Pathol Oral Radiol 2016;122(2): 143-151. DOI: 10.1016/j.000o.2016.03.004.

12. Ko EW-C, Lin $\mathrm{C}-\mathrm{H}$, Chen $\mathrm{Y}-\mathrm{A}$, et al. Enhanced surgical outcomes in patients with skeletal class iii facial asymmetry by 3-dimensional surgical simulation. J Oral Maxillofac Surg 2018;76(5):1073-1083. DOI: 10.1016/j.joms.2017.09.009.

13. Wolford LM, Chemello PD, Hilliard FW. Occlusal plane alteration in orthognathic surgery. J Oral Maxillofac Surg 1993;51(7):730-740. DOI: 10.1016/S0278-2391(10)80410-0.

14. Chemello PD, Wolford LM, Buschang PH. Occlusal plane alteration in orthognathic surgery--Part II: Long-term stability of results. Am J Orthod Dentofacial Orthop 1994;106(4):434-440. DOI: 10.1016/ S0889-5406(94)70066-4.

15. Reyneke JP, Bryant RS, Suuronen R, et al. Postoperative skeletal stability following clockwise and counter-clockwise rotation of the maxillomandibular complex compared to conventional orthognathic treatment. Br J Oral Maxillofac Surg 2007;45(1):56-64. DOI: 10.1016/j. bjoms.2005.12.015.

16. Mehra P, Downie M, Pita MC, et al. Pharyngeal airway space changes after counterclockwise rotation of the maxillomandibular complex. Am J Orthod Dentofacial Orthop 2001;120(2):154-159. DOI: 10.1067/ mod.2001.114647.

17. Brevi BC, Toma L, Pau M, et al. Counterclockwise rotation of the occlusal plane in the treatment of obstructive sleep apnea syndrome. J Oral Maxillofac Surg 2011;69(3):917-923. DOI: 10.1016/ j.joms.2010.06.189. 